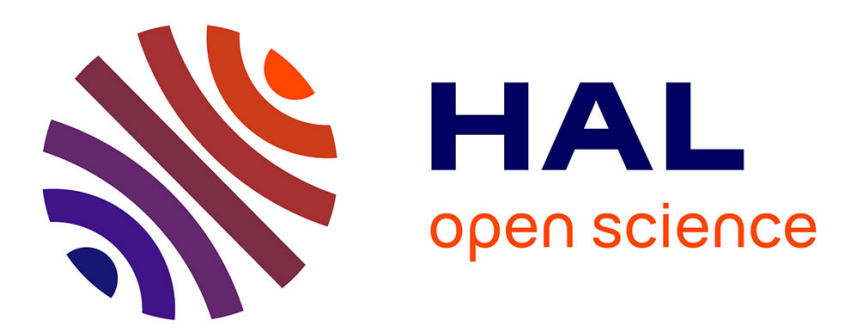

\title{
Rapid additive manufacturing of optimized anisotropic metaporous surfaces for broadband absorption
}

Théo Cavalieri, Jean Boulvert, Vicente Romero-García, Gwenael Gabard, Marie Escouflaire, Josselin Regnard, Jean-Philippe Groby

\section{- To cite this version:}

Théo Cavalieri, Jean Boulvert, Vicente Romero-García, Gwenael Gabard, Marie Escouflaire, et al.. Rapid additive manufacturing of optimized anisotropic metaporous surfaces for broadband absorption. Journal of Applied Physics, 2021, 129 (11), pp.115102. 10.1063/5.0042563 . hal-03436126

\author{
HAL Id: hal-03436126 \\ https://hal.science/hal-03436126
}

Submitted on 22 Nov 2021

HAL is a multi-disciplinary open access archive for the deposit and dissemination of scientific research documents, whether they are published or not. The documents may come from teaching and research institutions in France or abroad, or from public or private research centers.
L'archive ouverte pluridisciplinaire HAL, est destinée au dépôt et à la diffusion de documents scientifiques de niveau recherche, publiés ou non, émanant des établissements d'enseignement et de recherche français ou étrangers, des laboratoires publics ou privés. 


\section{Rapid additive manufacturing of optimised anisotropic metaporous surfaces for broadband absorption}

Théo Cavalieri, ${ }^{1,2, a)}$ Jean Boulvert, ${ }^{1,2,3}$ Vicent Romero-García, ${ }^{1}$ Gwénaël Gabard, ${ }^{1}$ Marie Escouflaire, ${ }^{2}$ Josselin Regnard, ${ }^{2}$ and Jean-Philippe Groby ${ }^{1}$

1) Laboratoire d'Acoustique de l'Université du Mans (LAUM), UMR CNRS 6613, Institut d'Acoustique - Graduate School (IA-GS), CNRS, Le Mans Université, France

${ }^{2)}$ Safran Aircraft Engines, Rond Point René Ravaud - Réau, 77550 Moissy-Cramayel, France

3) Laboratoire d'Analyse Vibratoire et Acoustique, LAVA, Department of Mechanical Engineering, École Polytechnique de Montréal, P.O. Box 6079 Station Centre-ville, Montréal, Qubec H3C 3A7, Canada

(Dated: February 4, 2021) 
Porous materials and metamaterials play a key role in sound absorbing and insulation solutions in acoustics. With the growing interest in additive manufacturing techniques, recent work has focused on the printing of porous and resonant structures for acoustic purposes. Usual metaporous surfaces/interfaces are generally built by periodically inserting resonant elements in an existing porous layer. This complex manufacturing process can be significantly simplified by using additive manufacturing techniques, which also eases the design and optimisation of the metaporous surface. In this work, the acoustic properties of the metaporous surface are controlled by simple geometric parameters defining both the anisotropic porous layer and the shapes of the resonators. Hence, we focus on optimising split-ring resonators embedded in a micro-treillis porous layer, which are built in a single part using additive manufacturing techniques. A finite-element method together with the Bloch wave decomposition provides a numerical model used to predict the reflection and absorption coefficients under normal incidence. The geometric parameters of the anisotropic metaporous surface are then optimised by non-linear minimisation techniques to maximise acoustic absorption. An optimal metaporous surface is 3D printed by fused-deposition modeling and its acoustic properties are measured in an impedance tube. The measurements are in good agreement with the predicted optimal broadband absorption coefficient. This work demonstrates the benefits of additive manufacturing for designing metaporous acoustic surfaces.

PACS numbers: 61.43.Gt, 81.05.Rm, 46.40.Ff, 52.38.Dx, 43.20.Gp

a)Theo.Cavalieri@gmail.com; https://tcaval.vercel.app 


\section{INTRODUCTION}

Porous materials are commonly used in the context of noise reduction, particularly for sound absorption over wide frequency ranges ${ }^{1,2}$. When rigidly backed, porous layers provide their strongest absorption for frequencies higher than that of the so-called quarterwavelength resonance and perfect absorption is obtained when critical coupling occurs ${ }^{3}$. Although efficient for broadband absorption, the use of homogeneous porous layers implies bulky and heavy structures to cope with practical applications. The absorption efficiency of porous layers can be improved at lower frequencies by grading the layer ${ }^{4}$, or by exploiting the anisotropy of the material for diffuse-field absorption ${ }^{5}$, but they still do not display deep-subwavelength properties.

Deep-subwavelength properties are on the contrary the specific features of metamaterials or metasurfaces ${ }^{6-11}$. Nevertheless, deep-subwavelength perfect absorption of metamaterials is usually narrow band, because of the high quality factor of resonators at low frequencies. Detuned resonators ${ }^{12,13}$ are thus used to widen the absorption efficiency of metasurfaces, but they usually do not display absorption as broadband as porous layers.

Metaporous surfaces thus appear as an excellent compromise between the broadband efficiency of porous layer and the deep-subwavelength features of metasurfaces ${ }^{14}$. They consist in periodically embedding possibly resonant inclusions in a porous layer. Their absorbing properties rely on a complex balance between the thermal and viscous losses in the porous layer and in the resonant elements, the resonances of the inclusions ${ }^{15,16}$, and trapped modes ${ }^{14,17}$ that confine the energy between the inclusions and the rigid backing, and extension of the Wood anomaly to periodic layers ${ }^{14}$. Although the resonances of the inclusion lead to the enhancement of the porous layer absorption (irrespective of the frequency range), perfect absorption is limited to the viscous regime, except if the resonator is directly connected to the surrounding medium, i.e., the air half space ${ }^{16}$. Initially proposed for transmission problems ${ }^{18-20}$, metaporous surfaces are particularly efficient for subwavelength broadband absorption in reflection problems and have thus seen an increasing interest these last years ${ }^{21-23}$. They have been studied extensively and coupled with other resonant elements $^{24}$ or combined with poroelastic layers, thus enabling the use of elastic resonators ${ }^{25}$. Nevertheless, the manufacturing of metaporous surfaces is still tedious and requires inserting resonant elements in porous layer in which the volume of the resonator has been previously 
removed. The porous material should also be available, thus its properties are subjected to constraints and cannot be freely chosen.

Over the past decade, rapid additive manufacturing methods have made significant advances and complex structures can now be printed with ever finer levels of accuracy. In this article, we optimise a metaporous surface that is then manufactured by fused-deposition modelling (FDM). The complete metasurface (i.e. the porous layer with the embedded resonators) is described by a small number of geometric parameters and numerically modelled by a finite-element method (FEM). Fine-tuning both the micro-geometry of the porous layer and the macro-geometry of the resonant inclusions can lead to an optimal design that provides high levels of acoustic absorption over a chosen frequency range. While the macrogeometry of the metaporous surface is directly optimised, the micro-geometry of the porous layer is optimised via effective acoustic properties calculated using the two-scale asymptotic homogenisation theory ${ }^{26-28}$ applied to the representative unit-cell. The optimisation itself is performed using the downhill simplex method with a cost function that relies on the FEM model $^{29,30}$. The porous layer in which the inclusions are embedded is composed of a transverse isotropic porous material, which prevents the use of multiple-scattering theory (MST), as proposed initially ${ }^{18}$.

The present article is organised as follows: we first introduce the studied geometry and the manufacturing techniques in Sec. II. We then describe the solution procedures and the numerical methods used to model and optimise the metaporous surfaces in Sec. III. In Sec. IV the validation procedure with experimental techniques is presented. Finally, multiple numerical and experimental results are presented and compared in Sec. V. Internal acoustic fields are also provided to give more physical insight into the role of the anisotropy of the porous material in the resonator coupling. Additionally, detailed validation procedures of the numerical solutions are given in Appendix A.

\section{METAPOROUS SURFACE}

In this section, we introduce the metaporous surface and its geometry. The additive manufacturing technique used to produce the samples is then presented. 


\section{A. Description of the configuration}

The design of the metaporous surface is illustrated in Fig. 1. It consists in acoustically rigid 2D split-ring resonators (SRR) periodically embedded in a transverse isotropic porous material. Several split-rings are embedded in the porous layer so as to form a super-cell. The whole medium is described in a two-dimensional Cartesian coordinate system $\mathcal{R}=\left(O, \mathbf{e}_{1}, \mathbf{e}_{2}\right)$ with position vector $\mathbf{x}=\left(x_{1}, x_{2}\right) \in \mathbb{R}^{2}$. The metaporous surface has a thickness $L_{2}=25 \mathrm{~mm}$ in the normal direction $\mathbf{e}_{2}$ and is of infinite extent in the transverse direction $\mathbf{e}_{1}$. The resonant inclusions are placed periodically within the porous layer, with a regular spacing along $\mathbf{e}_{1}$. The periodic medium is described by its lattice constant (or periodicity) $L_{1}=84 \mathrm{~mm}$. This corresponds to twice the transverse dimension of the impedance tube used for the experimental validation presented in Sec. IV.

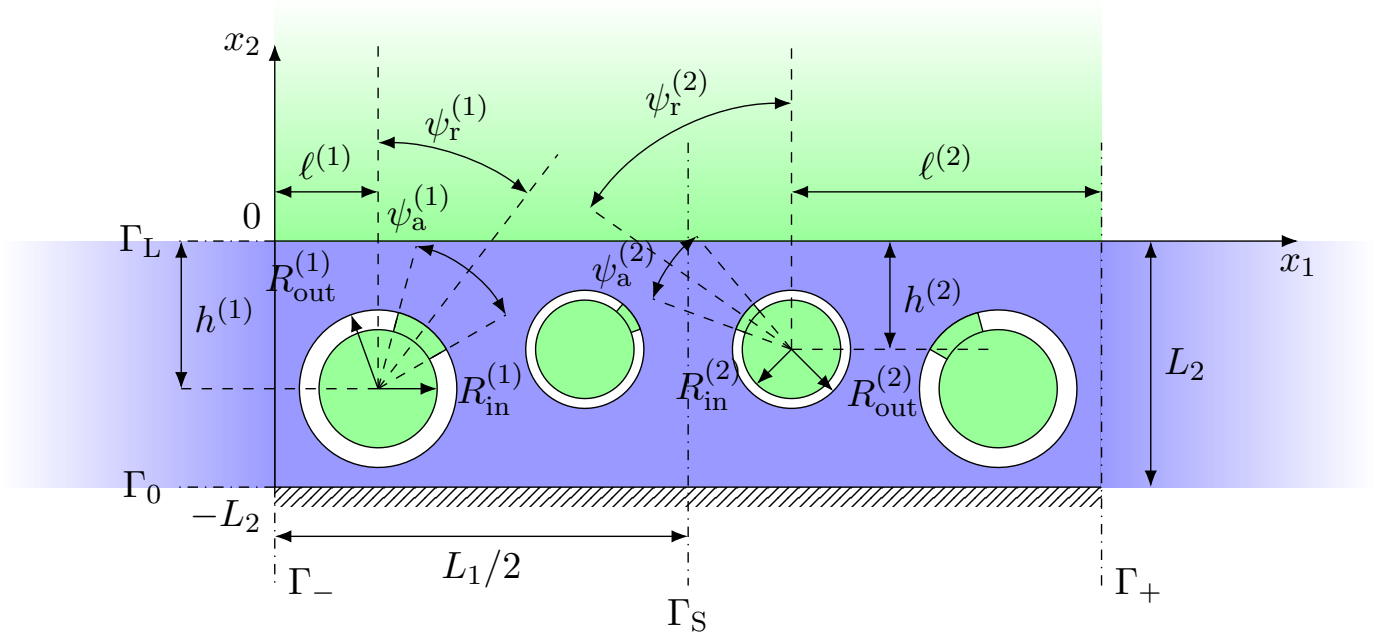

Figure 1. (Color online) Schematic of the split-ring resonators embedded in anisotropic porous material. In green is the air, in blue is the porous material $\Omega_{\mathrm{e}}$ with transverse isotropic properties, set between interfaces $\Gamma_{0}$ and $\Gamma_{\mathrm{L}}$, with periodic conditions on $\Gamma_{ \pm}$.

As shown in Fig. 1, four resonators are embedded within the porous material, which make up the periodic layer. The unit cell of this periodic medium is composed of two pairs of distinct resonators, and displays a reflection symmetry with respect to the vertical plane $\Gamma_{\mathrm{S}}$ along $\mathbf{e}_{2}$. Therefore, we will only focus on one half of the unit cell presented in Fig. 1. Each resonator $j \in \llbracket 1,2 \rrbracket$ is described by the following geometric parameters: the inner and outer radii $R_{\text {in }}^{(j)}$ and $R_{\text {out }}^{(j)}$ respectively, the depth $h^{(j)}$ (or center position along 
$\left.\mathbf{e}_{2}\right)$, the horizontal position $\ell^{(j)}=(2 j-1) L_{1} / 8$ and finally both aperture and rotation angles, respectively $\psi_{\mathrm{a}}^{(j)}$ and $\psi_{\mathrm{r}}^{(j)}$. In this work, the domains will be denoted as follows: the porous material is $\Omega_{\mathrm{e}}$, the $j$ th rigid resonator is $\Omega_{\mathrm{r}}^{(j)}$, the cavity of the $j$ th resonator is $\Omega_{\mathrm{c}}^{(j)}$, and the neck of the $j$ th resonator is $\Omega_{\mathrm{n}}^{(j)}$. The metaporous surface is then considered as $\Omega:=\left\{\mathbf{x}=\left(x_{1}, x_{2}\right) \mid \mathbf{x} \in \Omega_{\mathrm{e}} \bigcup_{j}\left(\Omega_{\mathrm{c}}^{(j)} \cup \Omega_{\mathrm{n}}^{(j)} \cup \Omega_{\mathrm{r}}^{(j)}\right)\right\}$.

\section{B. Manufacturing techniques}

Recent manufacturing techniques have been employed to build porous materials ${ }^{31,32}$. Such materials can exhibit anisotropic properties, which can play in our favour when looking for optimal structures. In order to manufacture such metasurfaces, we employ a fused-deposition modelling (FDM) technique. It consists in the deposition of molten polylactic acid (PLA), in the form of thin filaments. The raw material initially comes in the form of a solid wire of diameter $1.75 \mathrm{~mm}$. It is then heated to approximately $473 \mathrm{~K}$ within the nozzle, and deposited in thinner filaments of $0.4 \mathrm{~mm}$ in diameter. The structure is built layer after layer, which makes it possible to embed a rigid inclusion in the anisotropic porous material. To do so, the geometry of the optimal structure is first described in the standard tessellation language (STL). It is then translated in series of computer numerical control (CNC) instructions, known as G-code. This operation is readily performed by slicing softwares. The geometry of the porous material is controlled by the filling fraction $\mathcal{F}$ of the micro-trellis, which is a common infill pattern in slicing procedures. With a fixed filament diameter, the filling fraction is controlled by the lattice constant of the micro-trellis. The micro-trellis directly provides transverse isotropic properties, which can thus be tuned by changing the value of $\mathcal{F}$. 
(a)

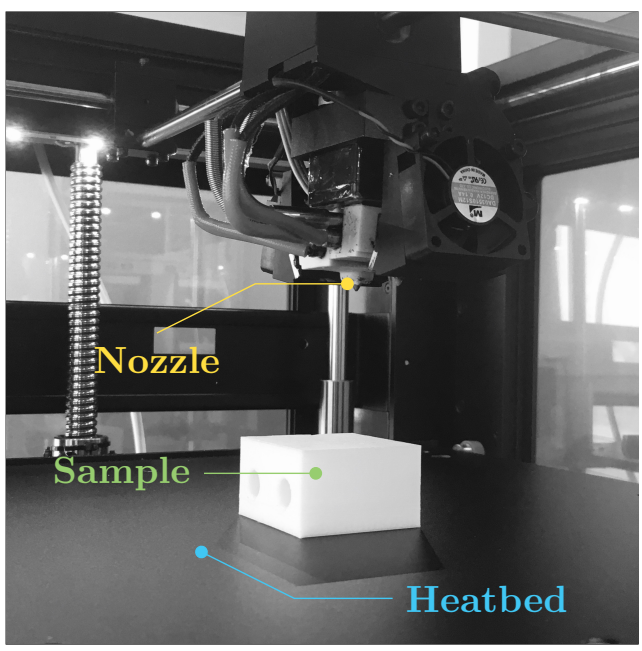

(c)

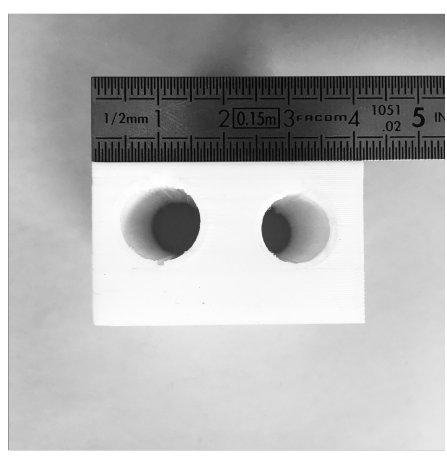

(b)

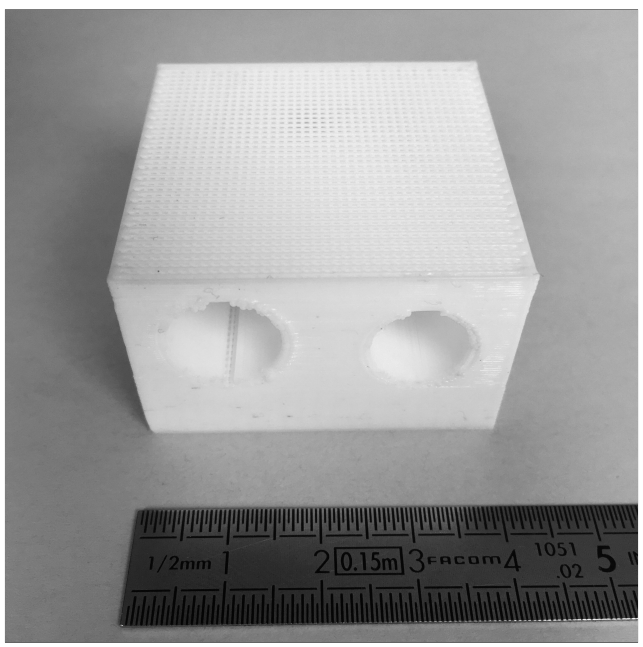

(e)

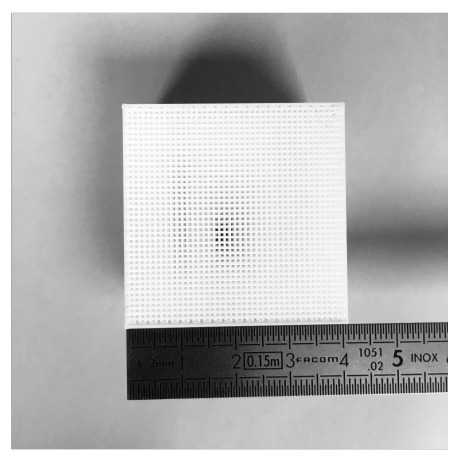

Figure 2. (Color online) (a) Photography of printing machine in operation. (b,c,d,e) Samples of the resonant porous structures. The red arrow in (e) represents the direction of incidence of the acoustic wave.

As seen in Fig. 2(a) the sample is placed on the heatbed under the nozzle. The cavities and necks of the resonators embedded in the porous layer can be seen in detail in Fig. 2 (b-e). In order to print the solid resonators, the filling fraction is set to $\mathcal{F}=1$. The metaporous super-cell is manufactured using the printer Raise3D Pro2. As the structure is build layer-by-layer, the actual manufactured geometry differs slightly from the theoretical geometry shown in Fig. 1. Optical microscopy is employed to provide qualitative analysis of the additive manufacturing defects. 
(a)

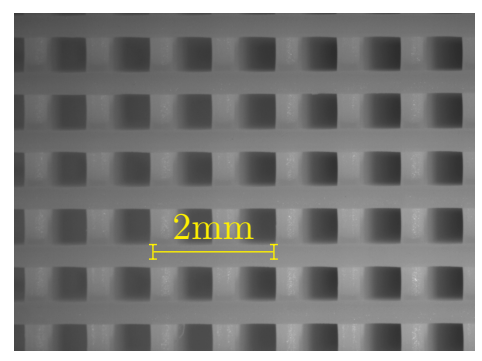

(b)

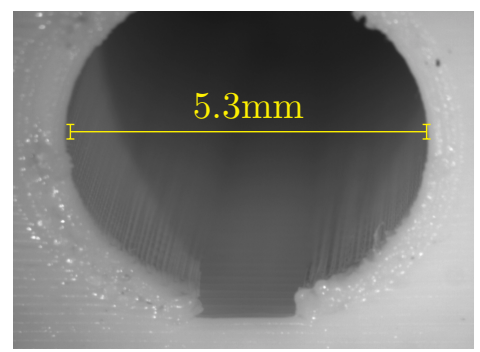

(c)

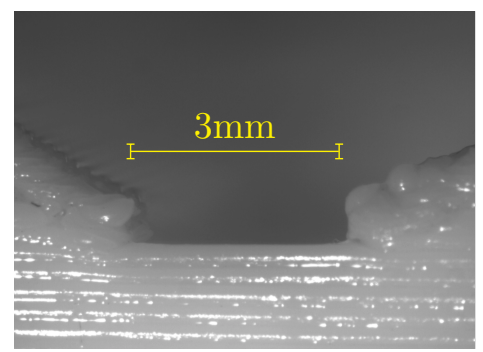

Figure 3. Optical microscopy images of (a) top-view the porous micro-structure, (b) the split-ring resonator and (c) side view of the SRR neck.

In Fig. 3(a) we observe the porous micro-trellis made of orthogonal filaments, with lattice constant $1 \mathrm{~mm}$. The porous material itself presents some defects, mainly the filament striction and surface roughness, which have been studied in previous works ${ }^{31}$. A crosssectional view of one of the SRRs is shown in Fig. 3(b), where we can observe surface roughness in the cavity of the resonator, linked to the layer-by-layer manufacturing process. This induced roughness was found to strongly affect the thermal and viscous losses when

of dimension comparable to the spacing between adjacent surfaces ${ }^{33}$. A close-up image of the SRR neck is shown in Fig. 3(c), illustrating the limits of FDM when dealing with parts of small dimensions. The combination of these discrepancies has an impact on the overall acoustic behaviour of the metaporous surface.

\section{MODELLING AND OPTIMISATION}

In order to optimise the metaporous surface, we need a predictive model for its acoustic properties. This is done using a finite-element method combined with the Bloch wave decomposition. The porous material is described as an equivalent fluid to account for viscous and thermal dissipation phenomena. The optimal geometric parameters are identified using a non-linear constrained minimisation of the reflection coefficient. 


\section{A. Equivalent fluid model}

The porous structure itself can be decomposed into two domains, $\Omega_{\mathrm{s}}$ and $\Omega_{\mathrm{f}}$ the solid and fluid phases, respectively. Hence, we denote $\Omega_{\mathrm{e}} \equiv \Omega_{\mathrm{f}} \cup \Omega_{\mathrm{s}}$ the total domain of the equivalent fluid, the open porosity of this two-phase medium is $\phi=\left|\Omega_{\mathrm{f}}\right| /\left|\Omega_{\mathrm{f}} \cup \Omega_{\mathrm{s}}\right|=1-\mathcal{F}$. As the propagation medium consists in a periodic arrangement of a micro-geometric unit cell, the proposed structure can be studied under scale separation ${ }^{26-28}$. At the microscopic scale (i.e. the scale of the micro-geometric unit cell), the rheology of the fluid phase is driven by thermo-acoustic and visco-inertial phenomena. Under a sufficiently large scale separation factor, the macroscopic effective properties of the medium can be described by homogenisation of the local fields. The Johnson-Champoux-Allard-Lafarge (JCAL) model gives an approximation for both thermal and viscous solutions ${ }^{34}$.

More recent works on additive manufacturing and sound propagation in graded porous layers have used the same description ${ }^{4,35,36}$. The aforementioned model relies on multiple parameters, which estimate the dynamic thermal and viscous permeabilities from an asymptotic description. Three of them are scalars $\left[\phi ; \Lambda^{\prime} ; \Theta^{0}\right]$ and related to thermal effects, respectively the open porosity, the thermal characteristic length and the thermostatic permeability. Three others are direction-dependent $\left[\tau_{j j}^{\infty} ; \Lambda_{j} ; K_{j j}^{0}\right]$ and related to viscous effects, respectively the high-frequency limit of tortuosity, the characteristic viscous length and the viscostatic permeability in the direction $\mathbf{e}_{j}$. Although the transport parameters can be estimated by FEM problems at the scale of the unit cell ${ }^{36,37}$, it has been shown that manufacturing defects play an important role in the measured effective properties ${ }^{31,33}$. In order to account for the internal geometrical defects, we rely on databases obtained from inverse characterisation techniques ${ }^{4,38}$. Thus, the JCAL parameters are obtained by fitting experimental results with respect to the filling fraction $\mathcal{F}$.

\section{B. Finite-Element Model}

We consider acoustic wave propagation under ambient conditions and in the linear harmonic regime, using the time convention $\mathrm{e}^{-\mathrm{i} \omega t}$. In the frequency domain, the homogeneous anisotropic Helmholtz equation in the two-dimensional Cartesian coordinate system is given 
by

$$
B \boldsymbol{\nabla} \cdot([\boldsymbol{H}] \boldsymbol{\nabla} p)+\omega^{2} p=0,
$$

with excess acoustic pressure $p$ and angular frequency $\omega$. The effective bulk modulus of the equivalent fluid is denoted $B$, and $[\boldsymbol{H}]=[\boldsymbol{\rho}]^{-1}$ is the inverse mass density tensor. These properties encapsulate the thermo-acoustic and visco-inertial properties of the equivalent fluid, and are complex and frequency-dependent functions.

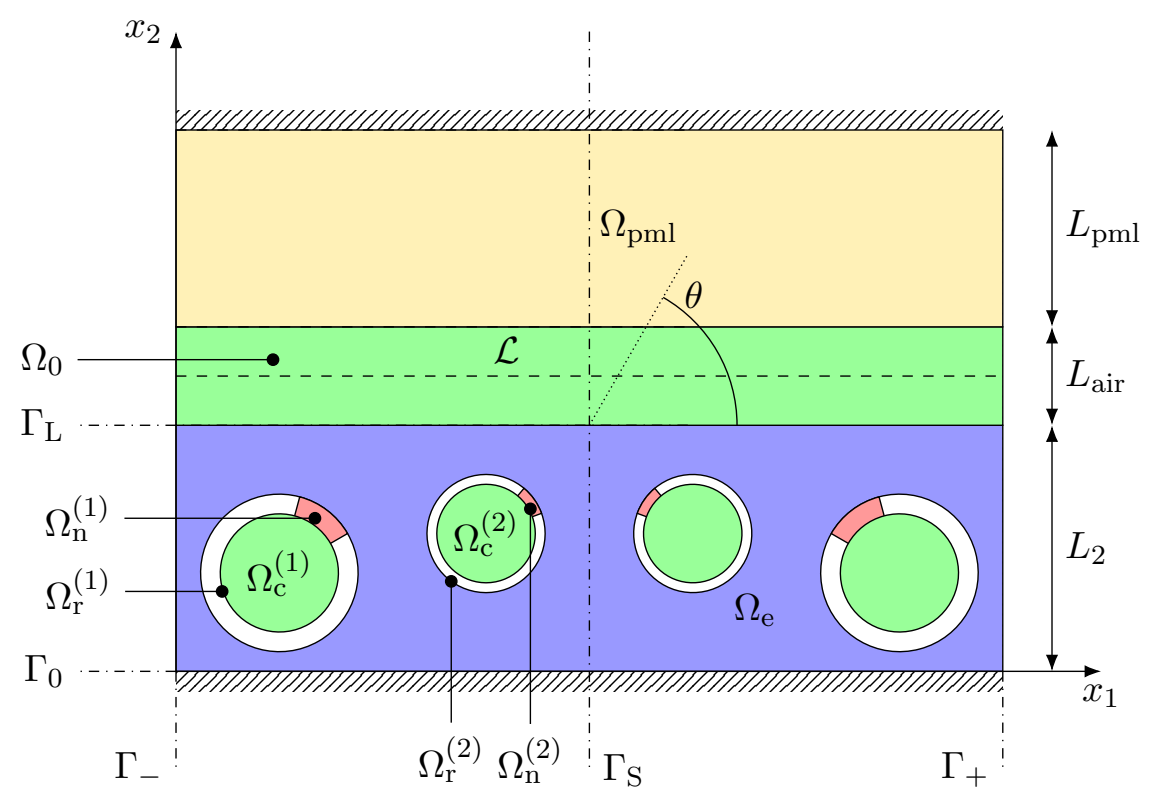

Figure 4. (Color online) Schematic of the finite-element model. The porous material is shown in blue, the air in green, the PML in yellow, the slits in red, and the rigid SRR in white. The interfaces are shown in dashdotted lines and the probing line-segment in dashed black line.

In the domain $\Omega_{0}$, we define an impinging plane wave in the form of

$$
p^{\mathrm{inc}}(\mathbf{x}, \omega)=|A| \mathrm{e}^{\mathrm{i} \varphi_{0}} \mathrm{e}^{\mathrm{i} \mathbf{k} \cdot \mathbf{x}}
$$

with the wavevector $\mathbf{k}=\left\{k_{1}, k_{2}\right\}^{T}$ where the superscript ${ }^{T}$ denotes the transposition. We have $k_{1}=k_{0} \sin (\theta)$ and $k_{2}=k_{0} \cos (\theta)$, with $k_{0}=\omega / c_{0}$ the wavenumber in free field, $c_{0}$ the speed of sound, and $\theta$ the angle of incidence with respect to $\mathbf{e}_{1}$. For simplicity, we set the amplitude $|A|=1$ and the phase offset $\varphi_{0}=0$. As the metaporous surface is rigidly backed, the normal particle velocity at the interface $\Gamma_{0}$ must be zero, so $\left.\mathbf{v} \cdot \mathbf{n}\right|_{\Gamma_{0}}=0$ where $\mathbf{v}$ is the vector of acoustic particle velocity. On the interface $\Gamma_{\mathrm{L}}$ between the porous layer and the ambient air, the excess pressure $p$ and the normal velocity $v_{2}$ are continuous. The 
periodicity of the acoustic field along $\mathbf{e}_{1}$ is enforced between the interfaces $\Gamma_{+}$and $\Gamma_{-}$by writing

$$
\left\{\begin{array}{l}
\left.p\right|_{\Gamma_{+}}=\left.p\right|_{\Gamma_{-}} \mathrm{e}^{\mathrm{i} \mathbf{k}_{\mathrm{B}} \cdot \mathbf{x}_{\mathrm{B}}} \\
\left.\mathbf{v} \cdot \mathbf{n}\right|_{\Gamma_{+}}=\left.\mathbf{v} \cdot \mathbf{n}\right|_{\Gamma_{-}} \mathrm{e}^{\mathrm{i} \mathbf{k}_{\mathrm{B}} \cdot \mathbf{x}_{\mathrm{B}}}
\end{array}\right.
$$

where the vector for Bloch periodicity is $\mathbf{k}_{\mathrm{B}}=\left\{k_{0} \sin (\theta), 0\right\}^{T}$ and $\mathbf{x}_{\mathrm{B}}=\left.\mathbf{x}\right|_{\Gamma_{+}}-\left.\mathbf{x}\right|_{\Gamma_{-}}$. The Sommerfeld radiation condition ${ }^{39,40}$ is given by

$$
\lim _{x_{2} \rightarrow+\infty} x_{2}^{1 / 2}\left(\frac{\partial}{\partial x_{2}}-\mathrm{i} k_{0}\right) p_{\mathrm{s}}\left(x_{2}\right)=0,
$$

so that the scattered pressure $p_{\mathrm{s}}$ vanishes far from the interface $\Gamma_{\mathrm{L}}$. This is implemented under the form of a perfectly matched layer $(\mathrm{PML})^{41}$. In the domains $\Omega_{\mathrm{n}}^{(j)}$, we model the thermal and viscous losses in the necks of the resonators. The necks are seen as slits of

dimension $d^{(j)}=2 R_{\text {in }}^{(j)} \sin \left(\psi_{\mathrm{a}}^{(j)} / 2\right)$, a chord on the $j$ th circle of inner radius $R_{\text {in }}^{(j)}$ and aperture angle $\psi_{\mathrm{a}}^{(j)}$, see Fig. 1. Assuming $d^{(j)} \ll \lambda$ in the slits, the acoustic wave propagation is described by effective parameters, namely the mass density $\rho_{\mathrm{n}}$ and characteristic impedance $Z_{\mathrm{n}}$ which are both complex-valued and frequency-dependent ${ }^{42}$. The air and PML domains $\Omega_{0}$ and $\Omega_{\mathrm{pml}}$ are meshed using quadrilateral elements while the porous layer, the necks and cavities are meshed with triangular elements. In order to capture finer details of the geometry, we use a total of $20 \times 10^{3}$ elements so that no element is larger than a fifth of the smallest dimension.

\section{Numerical estimation of reflection and absorption coefficients}

The scattered pressure field emerging from the interaction with the metaporous surface is quantified by its reflection coefficient. The following procedure enables the calculation of the reflection coefficient from a Bloch wave decomposition. As the scattered pressure field is periodic along the direction $x_{1}$, it is expressed as a sum of Bloch waves:

$$
p_{\mathrm{s}}(\mathbf{x}, \omega)=|A| \mathrm{e}^{\mathrm{i} \varphi_{0}} \sum_{q \in \mathcal{Q}} R_{q} \mathrm{e}^{\mathrm{i} \mathbf{k}^{(q)} \cdot \mathbf{x}},
$$

where the Bloch wave number $q$ is an integer belonging to $\mathcal{Q}=\llbracket-Q, Q \rrbracket \subset \mathbb{Z}$. The components of the wavevector $\mathbf{k}^{(q)}$ are given along both directions and for each value of $q$ by

$$
\mathbf{k}^{(q)}=\left\{\begin{array}{l}
k_{1}+2 \pi q / L_{1} \\
{\left[k_{0}^{2}-\left(k_{1}^{(q)}\right)^{2}\right]^{1 / 2}}
\end{array}\right\} .
$$


The coefficient $R_{q}$ corresponds to the reflection coefficient of the $q$ th Bloch wave. The scattered pressure field that emerges is numerically calculated at every point of the domain $\Omega_{0}$. The pressure field is then recorded on the line $\mathcal{L}$ shown in Fig. 4 and defined by $x_{2}=L_{2}+L_{\text {air }} / 2$ and $0<x_{1}<L_{1}$. The Bloch modes satisfy the following orthogonality relation $\forall\left(q, q^{\prime}\right) \in \mathcal{Q}^{2}$ :

$$
\int_{\mathcal{L}} \mathrm{e}^{\mathrm{i}\left(k_{1}^{(q)}-k_{1}^{\left(q^{\prime}\right)}\right) x_{1}} \mathrm{~d} x_{1}=|\mathcal{L}| \delta_{q, q^{\prime}},
$$

where $\delta_{q, q^{\prime}}$ is the Kronecker delta and $|\mathcal{L}|=L_{1}$. The reflection coefficient of the $q$ th Bloch wave is given by

$$
R_{q}=\frac{1}{|\mathcal{L}|} \mathrm{e}^{-\mathrm{i} k_{2}^{(q)} x_{2}(\mathcal{L})} \int_{\mathcal{L}} p_{\mathrm{s}}(\mathbf{x}, \omega) \mathrm{e}^{-\mathrm{i} k_{1}^{(q)} x_{1}} \mathrm{~d} x_{1}
$$

and the modulus of the total reflection coefficient reads

$$
|R(\omega)|^{2}=\sum_{q \in \mathcal{Q}}\left|R_{q}\right|^{2} \operatorname{Re}\left(k_{2}^{(q)}\right) / k_{2} .
$$

The acoustic absorption coefficient, which represents the fraction of energy dissipated within the metaporous surface, is given by

$$
\alpha(\omega)=1-|R(\omega)|^{2} \in \mathbb{R} .
$$

\section{Optimisation routines}

In order to optimise the geometric parameters of the metaporous surface, a constrained non-linear minimisation routine is employed. We use the Nelder-Mead (or downhill simplex) $\operatorname{method}^{29,30}$ to look for the optimal design. From an initial set of geometric parameters $\mathcal{P}_{0}$, the method takes a series of steps to minimise a cost function. The scheme stops when the convergence is reached (with respect to absolute tolerances), or when the maximum number of iterations is attained. The necks of the SRRs are chosen to be in opposite directions along $\mathbf{e}_{2}$ with $\psi_{\mathrm{r}}^{(1)}=0 \mathrm{rad}$ and $\psi_{\mathrm{r}}^{(2)}=\pi \mathrm{rad}$. Also, the center position $h^{(j)}$ of each resonator on the vertical axis $\mathbf{e}_{2}$ is dependent on the resonator's outer radius. In order to easily manipulate this geometric constraint, we introduce the relative depth $\bar{h}^{(j)}$, such as $h^{(j)}=R_{\text {out }}^{(j)}+\bar{h}^{(j)}\left(L_{2}-2 R_{\text {out }}^{(j)}\right)$. The vector of parameters to be optimised is written

$$
\mathcal{P}:=\left\{\mathcal{F}, \psi_{\mathrm{a}}^{(1)}, \psi_{\mathrm{a}}^{(2)}, R_{\mathrm{in}}^{(1)}, R_{\text {in }}^{(2)}, R_{\text {out }}^{(1)}, R_{\text {out }}^{(2)}, \bar{h}^{(1)}, \bar{h}^{(2)}\right\}^{T} \subset \mathbb{R}^{9}
$$


the bounds of which are described by $\mathcal{P}_{ \pm}$:

$$
\left\{\begin{array}{l}
\mathcal{F} \in] 0,1[ \\
\psi_{\mathrm{a}}^{(j)} \in[0, \pi / 2] \\
R_{\text {in }}^{(j)} \in\left[1 \times 10^{-3}, \max \left(L_{1} / 8, L_{2} / 2\right)\right] \\
R_{\text {out }}^{(j)} \in\left[1 \times 10^{-3}, \max \left(L_{1} / 8, L_{2} / 2\right)\right] \\
\bar{h}^{(j)} \in[0.1,0.9]
\end{array}\right.
$$

Moreover, some constraints are imposed over the geometric parameters, in order to satisfy the overall topology of the system shown in Fig. 1. The constraints are established as the outer perimeter of the resonator is contained in $\Omega_{\mathrm{e}}$, and the wall thickness of the resonators must be greater than 800 microns. This translates into

$$
\left\{\begin{array}{l}
\forall j=\llbracket 1,2 \rrbracket \\
R_{\text {out }}^{(j)}-R_{\mathrm{in}}^{(j)} \geq 8 \times 10^{-4} \\
R_{\mathrm{out}}^{(j)} \leq \min \left(L_{1} / 8, L_{2} / 2\right) .
\end{array}\right.
$$

The acoustic absorption coefficient is maximised within a certain frequency range, when the cost function gets closer to zero. In order to do so, we define a cost function that translates how far the current vector of parameters is from the optimal value $\alpha=1$. The cost function is the arithmetic average of the absorption within the frequency range:

$$
\left.J(\mathcal{P}):=1-\frac{1}{N_{\mathrm{f}}} \sum_{i=1}^{N_{\mathrm{f}}} \alpha\left(f_{i}\right) \in\right] 0,1[
$$

with $N_{\mathrm{f}}$ the number of frequencies. The minimisation procedure requires an initial guess, which is chosen by setting the vector $\mathcal{P}_{0}$ with random parameters, following the uniform

distribution $\mathcal{P}_{0}^{(i)}:=\mathcal{U}\left(\mathcal{P}_{-}^{(i)}, \mathcal{P}_{+}^{(i)}\right)$. In this work, we focus on the frequency range between $f_{\min }=1000 \mathrm{~Hz}$ and $f_{\max }=4000 \mathrm{~Hz}$, with $N_{\mathrm{f}}=10$.

\section{EXPERIMENTAL SETUP}

As shown in Fig. 1 the unit cell of the periodic system is symmetric, hence the structure behaves as if half the unit cell was bounded by rigid walls (acting as perfect mirrors) when excited by an plane wave at normal incidence ${ }^{43}$. Thus, the Bloch modes on the total unit cell are identical to those within rigid boundaries assuming one half of the symmetric unit 
cell is placed in an impedance tube. In this section, we describe the experimental setup where the proposed metaporous surface is rigidly backed in an impedance tube. We give details on the collecting and processing of experimental data used to retrieve the absorption coefficient.

\section{A. Impedance tube measurement}

The waveguide has a square cross-sectional area of $42 \times 42 \mathrm{~mm}^{2}$ and is made of aluminum. The cut-off frequency of the first transverse mode is thus $f_{\mathrm{c}}=c_{0} / 0.084 \approx 4060 \mathrm{~Hz}$. A copper plug is backing the metaporous surface at position $x_{2}=-L_{2}$ ensuring the Neumann boundary condition.

The pressure field is evaluated at $N=11$ non-equidistant points located along $x_{2}$. We use a GRAS 46BD 1/4" CCP Pressure Standard Microphone Set, together with the conditioner GRAS Power Module Type 12AQ. The microphone is moved along the $\mathbf{e}_{2}$ axis, driven by the stepping motor Nanotec TYPE 4H8618M1408, and controlled using CHARLYROBOT F4350 CERNEX.

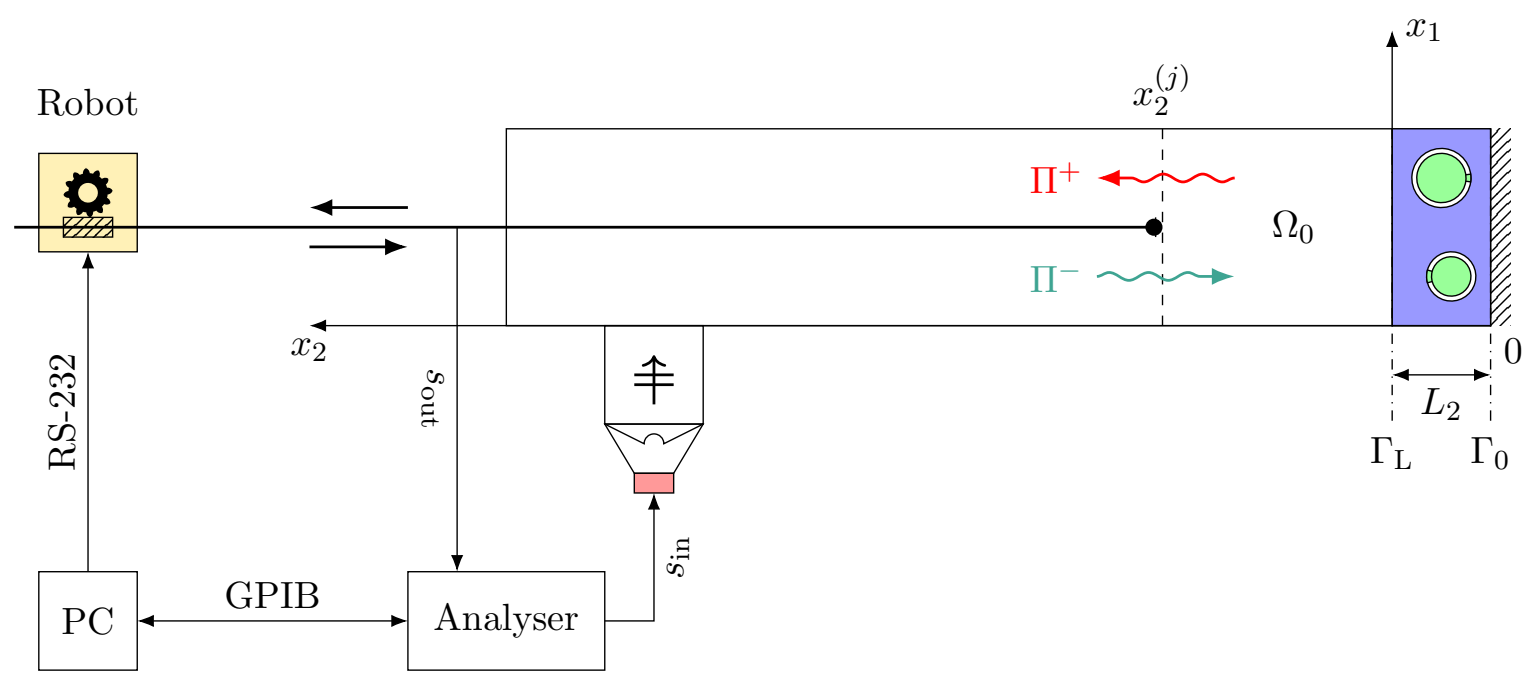

Figure 5. (Color online) Schematic of the experimental setup.

The frequency range is spanned linearly with the emission of $N_{\mathrm{f}}=200$ monochromatic plane waves. The pressure field is averaged over $M=2^{9}=512$ periods, where the period is $T=2 \pi / \omega$. We use the analyser Stanford SRS MODEL SR785 for the input electrical signal, which is finally transformed into a plane wave acoustic field by a loudspeaker. Since the rod 
used to move the microphone is at the end of the tube, the source is positioned to the side as shown in Fig. 5. This change is not of predominant importance as only low-frequency plane-waves are considered, below the cut-off frequency of the tube.

\section{B. Data processing}

For frequencies below the cut-off frequency $f_{\mathrm{c}}$, the pressure at point $j$ and angular frequency $\omega$ is the sum of the forward and backward plane waves:

$$
p_{j}(\omega)=\Pi^{+}(\omega) \mathrm{e}^{\mathrm{i} k_{0} x_{2}^{(j)}}+\Pi^{-}(\omega) \mathrm{e}^{-\mathrm{i} k_{0} x_{2}^{(j)}}
$$

where $\Pi^{ \pm}$are the complex amplitudes of the forward and backward propagating waves. The measurements at the different points $j$ can be combined in matrix form:

$$
\left\{\begin{array}{c}
p_{1} \\
p_{2} \\
\vdots \\
p_{N}
\end{array}\right\}=\left(\begin{array}{cc}
\mathrm{e}^{\mathrm{i} k_{0} x_{2}^{(1)}} & \mathrm{e}^{-\mathrm{i} k_{0} x_{2}^{(1)}} \\
\mathrm{e}^{\mathrm{i} k_{0} x_{2}^{(2)}} & \mathrm{e}^{-\mathrm{i} k_{0} x_{2}^{(2)}} \\
\vdots & \vdots \\
\mathrm{e}^{\mathrm{i} k_{0} x_{2}^{(N)}} & \mathrm{e}^{-\mathrm{i} k_{0} x_{2}^{(N)}}
\end{array}\right)\left\{\begin{array}{c}
\Pi^{+}(\omega) \\
\Pi^{-}(\omega)
\end{array}\right\} \Leftrightarrow \mathbf{p}=[\mathcal{E}] \boldsymbol{\Pi} .
$$

The complex amplitudes $\Pi^{+}(\omega)$ and $\Pi^{-}(\omega)$ can be retrieved by inverting the system of Eq. (16),

$$
\Pi=[\tilde{\mathcal{E}}] \mathbf{p}
$$

As the system is of dimension $\operatorname{dim}[\mathcal{E}]=2 \times N$, its pseudo-inverse is computed using the generalised Moore-Penrose inverse ${ }^{44,45}$. It is based on the singular value decomposition $(\mathrm{SVD})^{30}$ so that $[\tilde{\mathcal{E}}]=\mathbf{V}[\boldsymbol{S}]^{-1} \mathbf{U}^{\star}$, where $[\boldsymbol{S}]$ is the matrix of singular values, $[\boldsymbol{U}]$ and $[\boldsymbol{V}]$ the left- and right-singular vectors respectively. The superscripts ${ }^{\star}$ and ${ }^{\sim}$ denote the complex conjugation and pseudo-inverse respectively. The complex reflection coefficient is obtained from the ratio of the two complex amplitudes:

$$
R(\omega)=\Pi^{+} / \Pi^{-} \in \mathbb{C},
$$

and the absorption coefficient is given by Eq. (10).

\section{RESULTS AND DISCUSSIONS}

In this section, results are presented in terms of absorption, comparing numerical predictions with experimental data. Moreover, the internal acoustic fields for the pressure $p$ and 
particle velocity $\mathbf{v}$ are discussed based on the FEM simulations.

\section{A. Maximised broadband absorption}

We optimise the aforementioned macro- and micro-geometries, as defined by Eq. (11), so as to maximise the acoustic absorption between $f_{\min }$ and $f_{\max }$. The predicted and measured acoustic absorption of the optimal metaporous surface are given in Fig. 6(c). For comparison, we also show the absorption results for two other cases: first, the porous material alone in the absence of inclusion, and a case where the SRRs are replaced by rigid circular inclusions (non-resonant). These configurations are investigated numerically and experimentally in Fig. 6(a) and Fig. 6(b) respectively. The geometric parameters of the optimised metaporous surface are given in Tab. I.

(a)

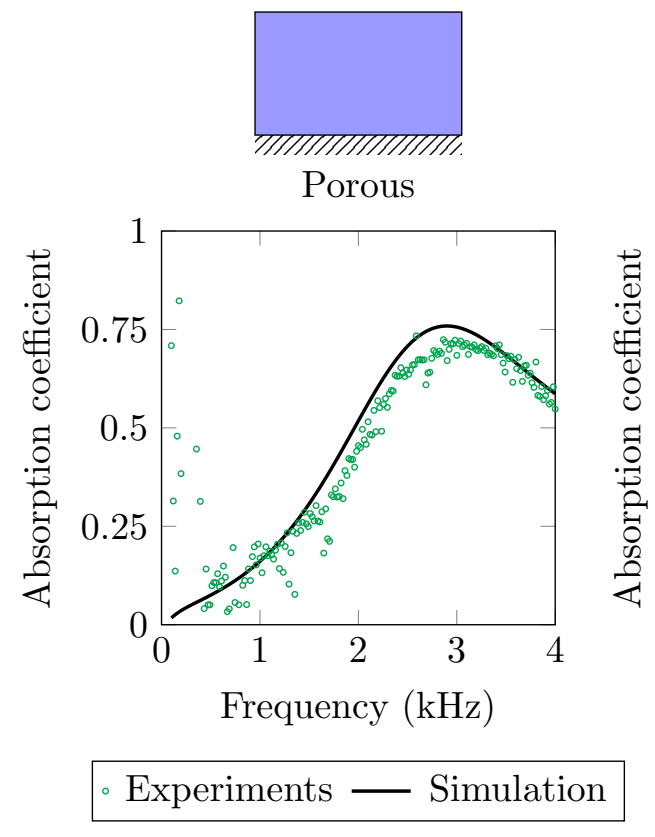

(b)
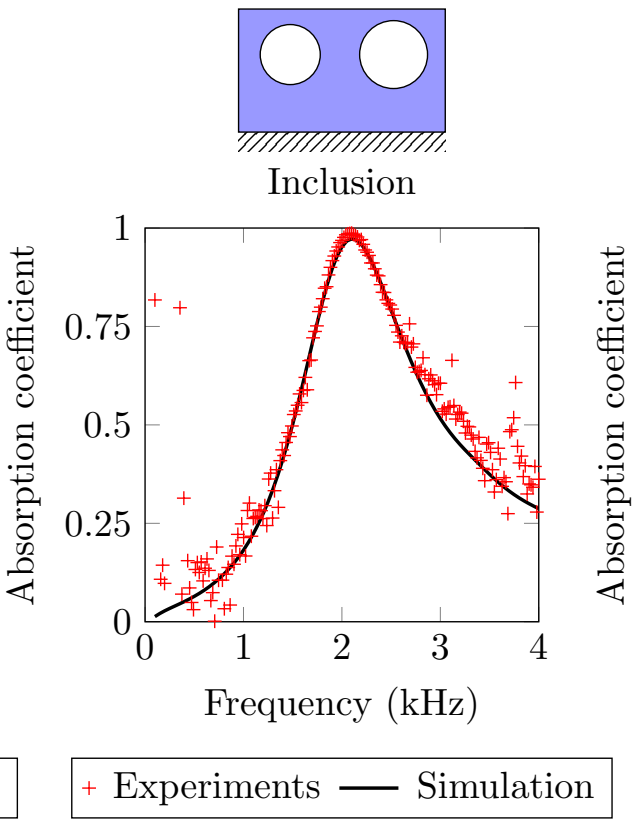

(c)
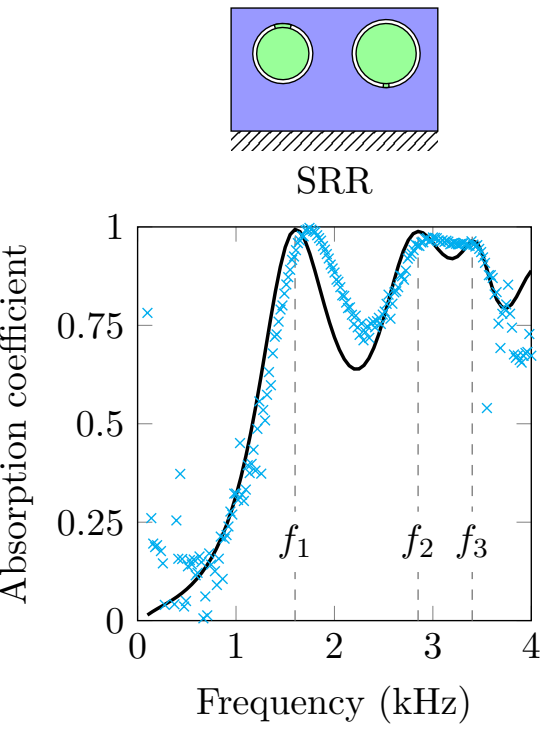

Experiments — Simulation

Figure 6. Measured and predicted absorption coefficients. (a) Porous material alone, (b) porous material with circular inclusions, (c) porous material with SRR.

Looking at Fig. 6(a,b), the experimental results and simulated predictions are in very good agreement. We notice small discrepancies which can emerge from a combination of distinct phenomena in Fig. 6(c). The viscous and thermal losses inside the SRR cavities are 
not accounted for in the numerical simulations, and as discussed in Sec. II the manufacturing process induces defects to the micro-structure of the porous material and to the geometry of the resonators. When the physics of the system is driven at the local scale (in the presence of SRR), it is very sensitive to small manufacturing defects. This is the reason why the discrepancies are much more visible in Fig. 6(c) for the SRR, than in Fig. 6(a,b) for the porous layer and the non-resonant inclusions.

\begin{tabular}{ccccccccc}
\hline $\mathcal{F}$ & $\psi_{\mathrm{a}}^{(1)}(\mathrm{deg})$ & $\psi_{\mathrm{a}}^{(2)}(\mathrm{deg})$ & $R_{\mathrm{in}}^{(1)}(\mathrm{mm})$ & $R_{\mathrm{in}}^{(2)}(\mathrm{mm})$ & $R_{\text {out }}^{(1)}(\mathrm{mm})$ & $R_{\text {out }}^{(2)}(\mathrm{mm})$ & $\bar{h}^{(1)}$ & $\bar{h}^{(2)}$ \\
\hline \hline 0.40 & 33 & 10 & 5.3 & 6.1 & 6.1 & 6.9 & 0.35 & 0.21 \\
\hline
\end{tabular}

Table I. Geometric parameters of the optimised metaporous surface.

Experimentally the system with non-resonant circular inclusions reaches quasi-perfect absorption $\alpha \approx 0.985$ at the frequency $f=(2100 \pm 20) \mathrm{Hz}$ in Fig. 6(b). This absorption peak reveals the presence of a trapped mode that confines the acoustic energy between the rigid backing and the periodic inclusions. For the SRR configuration in Fig. 6(c), three distinct peaks of absorption are observed. Over the frequency range of optimisation, we experimentally reach a mean absorption of $\alpha_{\text {avg }} \approx 0.82$, with quasi-perfect absorption $\alpha \geq 0.999$ at $f=(1720 \pm 20) \mathrm{Hz}$. This corresponds to a sub-wavelength regime as $L \approx \lambda / 8$. To gain more insight into the physical mechanisms responsible for these three peaks, it is useful to analyse the corresponding fields of acoustic pressure and particle velocity provided by the FEM model and presented in Fig. 7.

\section{B. Internal fields and couplings}

When excited at normal incidence and in the absence of inclusion, the transverse particle velocity $v_{1}$ is zero. In this case, only the properties in the normal direction $\mathbf{e}_{2}$ of the equivalent fluid are contributing to the acoustic behaviour of the homogeneous porous layer. In our case, the periodic inclusions introduce resonances and trapped modes that couple the velocity field in both its normal and transverse directions. The FEM provides the complete solution to the anisotropic Helmholtz equation within the metaporous surface and one can retrieve the internal fields. We use these fields to better understand the behaviour of the metaporous surface illustrated in Fig. 6. More precisely, we focus on the first peaks at $f_{1}=(1700 \pm 50) \mathrm{Hz}$ 
and $f_{3}=(3400 \pm 50) \mathrm{Hz}$ which correspond to the resonances of the SRRs. The peak at $f_{2}=(2850 \pm 50) \mathrm{Hz}$ is the trapped mode which increases the energy located between the inclusions and the rigid backing. From the results in the aforementioned Fig. 6, the absolute scattered pressure $\left|p_{s}\right|$ is minimised in $\Omega_{0}$ at those frequencies (since the reflection is minimised). Along with the pressure field, the normal and transverse components of the acoustic particle velocity shown in Fig. 7 exhibit the couplings introduced by the SRRs. 

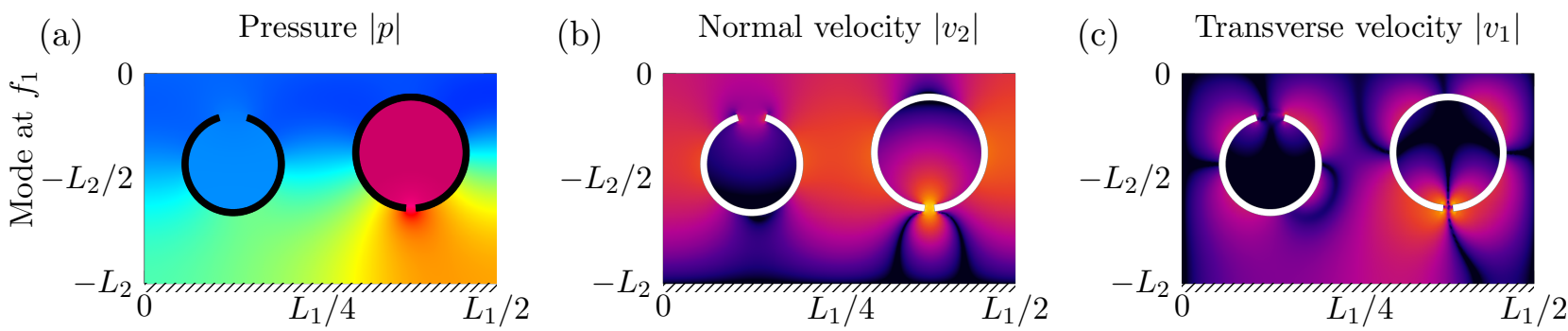

(d)

(e)

(f)
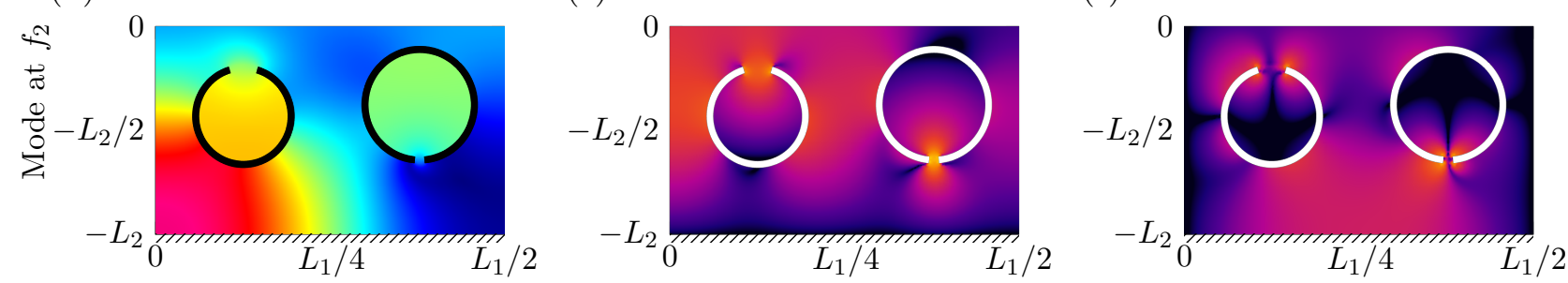

(g)

(h)
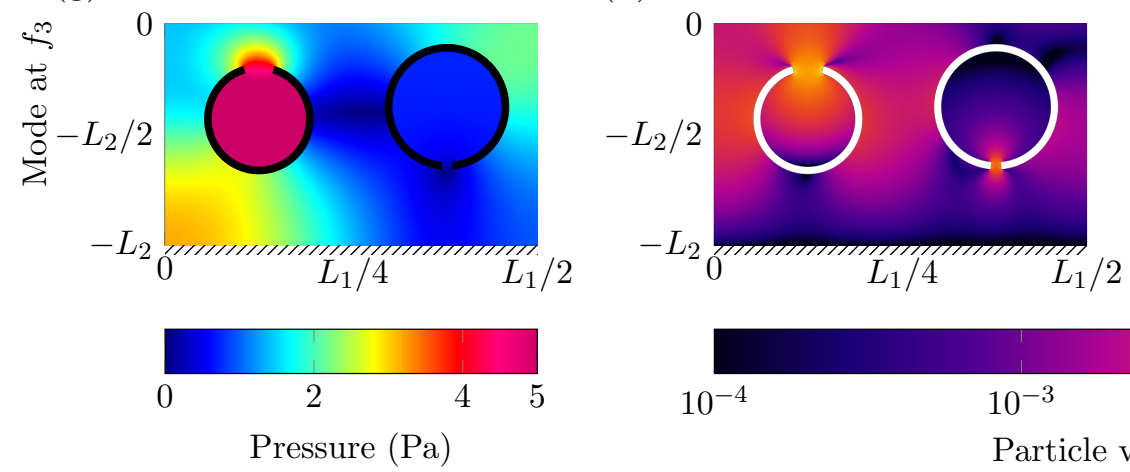

(i)
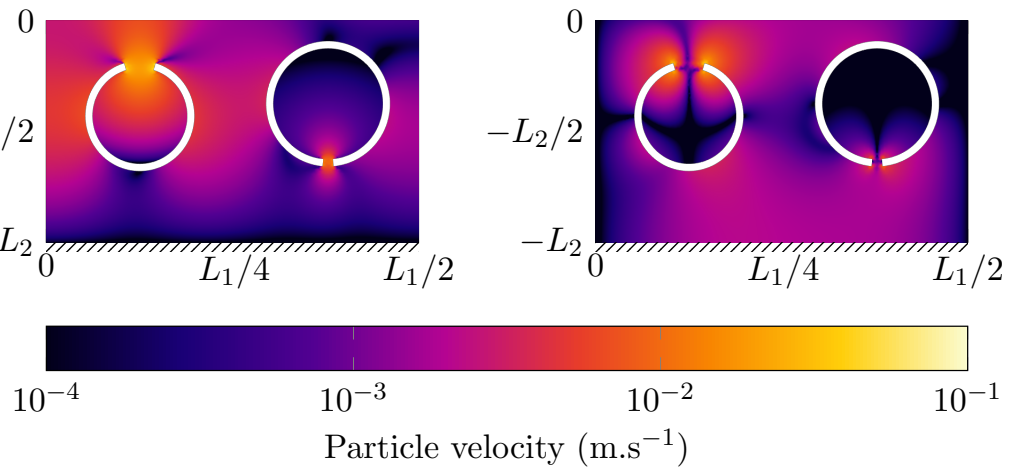

Figure 7. (Color online) Internal fields for acoustic pressure (a,d,g), transverse (b,e,h) and normal (c,f,i) particle velocity. Top row (a,b,c) shows the fields for the Mode 1 at $f=1650 \mathrm{~Hz}$, middle row (d,e,f) shows the fields at Mode 2, with $f=2850 \mathrm{~Hz}$ and bottom row (g,h,i) shows the fields at Mode $3, f=3400 \mathrm{~Hz}$. The colorbar on the bottom-left corner relates the pressure fields (a,d,g) while the colormap on the bottom-right corner is attributed to velocity fields (b,c,e,f,h,i). The latter is intentionally saturated as the lower bound is limited to three orders of magnitude below the maximal value of the field.

Regarding the mode at $f_{1}$, we see in Fig. $7($ a) that the largest values of pressure are confined within $\Omega_{\mathrm{c}}^{(2)}$, which correspond to the resonance of this SRR at that particular frequency. This resonance induces a strong particle velocity in the neck $\Omega_{\mathrm{n}}^{(2)}$ of the resonator, as shown in Fig. 7(b). At frequency $f_{2}$, the excess pressure is trapped between the rigid backing at $\Gamma_{0}$ and the rigid SRR $\Omega_{\mathrm{r}}^{(1)}$. This trapped mode is clearly visible on the pressure 
field in Fig. 7(d) as the strong values of pressure indicates the presence of this localised mode. For the mode at frequency $f_{3}$, we observe large values of pressure within the SRR cavity $\Omega_{\mathrm{c}}^{(1)}$ in Fig. $7(\mathrm{~g})$. Again, the high values of normal particle velocity in Fig. $7(\mathrm{~h})$ highlight this resonance phenomena at frequency $f_{3}$.

Although the metaporous surface is excited by a monochromatic plane wave at normal incidence, the resonators introduce a strong coupling in the porous domain $\Omega_{\mathrm{e}}$ between the normal and transverse directions. The Figs. 7(b,c,e,f,h,i) show the decimal logarithm of normal and transverse velocity fields within the metaporous surface. We notice that both transverse and normal velocity fields are of the same orders of magnitude, between $10^{-4} \mathrm{~m} . \mathrm{s}^{-1}$ and $10^{-1} \mathrm{~m} . \mathrm{s}^{-1}$. Thus, the normal and transverse properties of the equivalent fluid, which are encapsulated in the mass density tensor $[\boldsymbol{\rho}]$, are both contributing to the overall acoustic behaviour of the metaporous surface.

\section{CONCLUSIONS}

Porous materials and metamaterials are commonly used in acoustics, for absorption and insulation purposes. In this work, we propose an optimal design of resonant metaporous surface for acoustic absorption purposes. The proposed solution consists in the periodic embedding of multiple split-ring resonators in a transverse isotropic porous layer. By tuning the macro-geometry of rigid resonators as well as the micro-geometry of the porous medium, we maximise the absorption in the frequency range between 1 and $4 \mathrm{kHz}$. The metaporous surface provides strong broadband absorption capabilities and subwavelength quasi-perfect absorption.

The numerical simulations based on FEM provide a predictive model for the reflection and absorption coefficients. This predictive model is used in the cost function for the minimisation algorithm, whose goal is to maximise the absorption in a target range of frequencies.

Experiments have been conducted in an impedance tube with a square cross section. They provide a validation of the numerical model, as the experimental data are in good agreement with the simulated results. Additive manufacturing techniques have proved their ability to build resonant metaporous surfaces.

The present work demonstrates the capabilities of metaporous surfaces for absorbing sound waves, and highlights the use of additive manufacturing for such acoustic treatments. 
While the design and manufacturing of materials incorporating inclusions is usually tedious, the solutions presented here are fabricated in a single stage. In addition to offering a solution that is easy and quick to implement, this first prototype was developed at low cost, although this is slightly higher than for conventional porous materials.

Several points of this work provide perspectives for future work. Accounting for the oblique incidences would make it possible to obtain optimal structures for applications in diffuse acoustic fields, which however requires particular attention to the experimental validation. Finally, the optimisation of the shape and number of inclusions could offer more possibilities regarding the compromise between structural dimensions and frequency range of absorption.

\section{APPENDIX A: VALIDATION OF THE NUMERICAL MODEL}

The FEM model is compared to two other approaches in order to validate the simulation results when considering anisotropic porous materials and in presence of inclusions.

\section{A. Transfer matrix method}

The acoustic wave propagation in a homogeneous porous medium can be derived using the well known transfer matrix method $(\mathrm{TMM})^{46}$. Using the state-vector formalism, applied to the acoustic pressure and the normal component of velocity we introduce $\mathbf{W}=\left\{p, v_{2}\right\}^{T}$. The acoustic fields satisfy the following differential system of equations

$$
\partial_{x_{2}} \mathbf{W}-\mathrm{i} \omega\left[\begin{array}{cc}
0 & H_{22}^{-1} \\
B_{\text {eq }}^{-1} & 0
\end{array}\right] \mathbf{W}=0
$$

with $B_{\text {eq }}$ the equivalent bulk modulus, possibly accounting for out-of-plane rotations and oblique incidence. $H_{22}$ is the apparent inverse mass density in the normal direction ${ }^{35,36}$. As the normal particle velocity $v_{2}$ is imposed to be zero on the interface $\Gamma_{0}$, we have the boundary condition $\left.\mathbf{W}\right|_{\Gamma_{0}}=\{p, 0\}^{T}$. The state vector can then be calculated using matrix exponentials:

$$
\left\{\begin{array}{c}
1+R \\
(R-1) / \mathcal{Z}
\end{array}\right\}_{\Gamma_{\mathrm{L}}}=[\boldsymbol{M}]\left\{\begin{array}{c}
p(0) \\
0
\end{array}\right\}_{\Gamma_{0}},
$$


with $\mathcal{Z}=\left(\rho_{0} c_{0}\right) / \sin (\theta)$ and $[\boldsymbol{M}]$ the matricant ${ }^{47,48}$. From the differential system of equations, the reflection coefficient is obtained as

$$
R=\left(\frac{M_{11}}{M_{21}} \mathcal{Z}+1\right) /\left(\frac{M_{11}}{M_{21}} \mathcal{Z}-1\right)
$$

and the absorption coefficient is $\alpha=1-|R|^{2}$. The JCAL parameters of the homogeneous isotropic porous material are given in Tab. II.

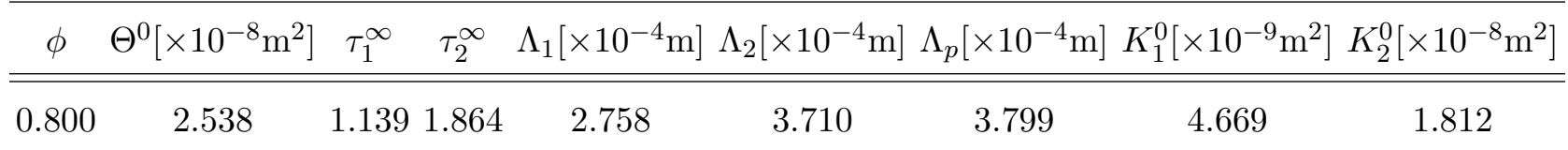

Table II. JCAL parameters of the homogeneous transverse isotropic porous material in the directions $\mathbf{e}_{1}$ and $\mathbf{e}_{2}$.

For this case of numerical validation, we consider a porous layer of thickness $L_{2}=25 \mathrm{~mm}$. The results in Fig. 8 show the comparison of FEM and TMM results for different angles of incidence.

(a)

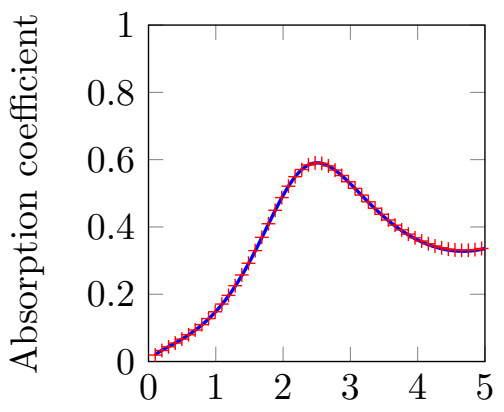

(c) Frequency $(\mathrm{kHz})$

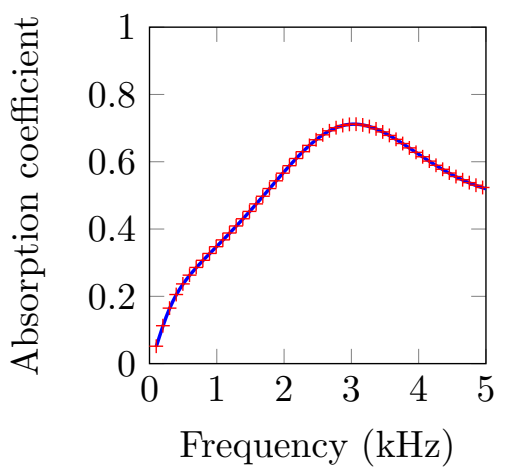

(b)

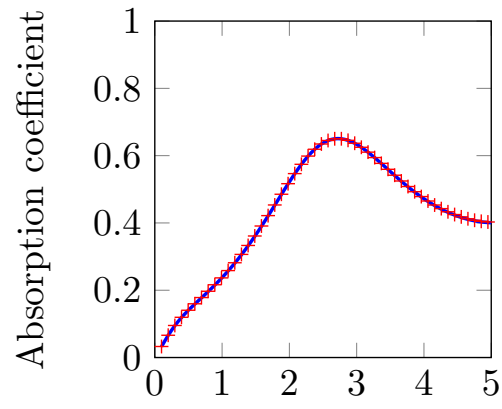

(d) Frequency $(\mathrm{kHz})$

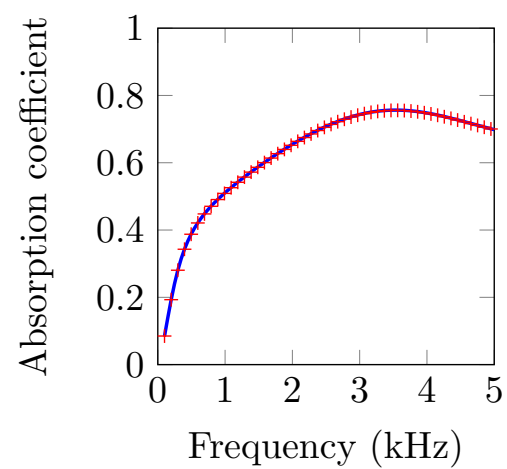

$\mathrm{TMM}+\mathrm{FEM}$

Figure 8. (Color online) Comparison of FEM and TMM results, for angles of incidence $\theta=\pi / 2$ (a), $\theta=\pi / 3(\mathrm{~b}), \theta=\pi / 4(\mathrm{c})$, and $\theta=\pi / 6(\mathrm{~d})$. 
The results are in excellent agreement, showing that both methods can be used to describe the propagation of waves in an homogeneous and anisotropic porous material.

\section{B. Multiple scattering theory}

In the particular case where the porous layer is isotropic and the inclusions are cylindrical, the multiple scattering theory (MST) provides an analytical description of the acoustic fields. As this theory is not the centrepiece of this work, it is only briefly presented in this appendix for validation purposes. The total acoustic field is a superposition of the undisturbed (meaning incident, in absence of inclusions) and scattered fields ${ }^{49}$. The scattered field emerges from the collective effect produced by the interactions of the undisturbed field with every obstacle. In response to the undisturbed acoustic excitation, every obstacle contributes to the global scattered field. For every obstacle, a local description of the scattered field in polar coordinates system leads to cylindrical wave functions, i.e. Bessel and Hankel functions. The Bloch wave decomposition provides a solution for the total field as previously explained in Sec. III. In order to compare the FEM results to the MST solutions, we consider a simple geometry shown in Fig. 9.

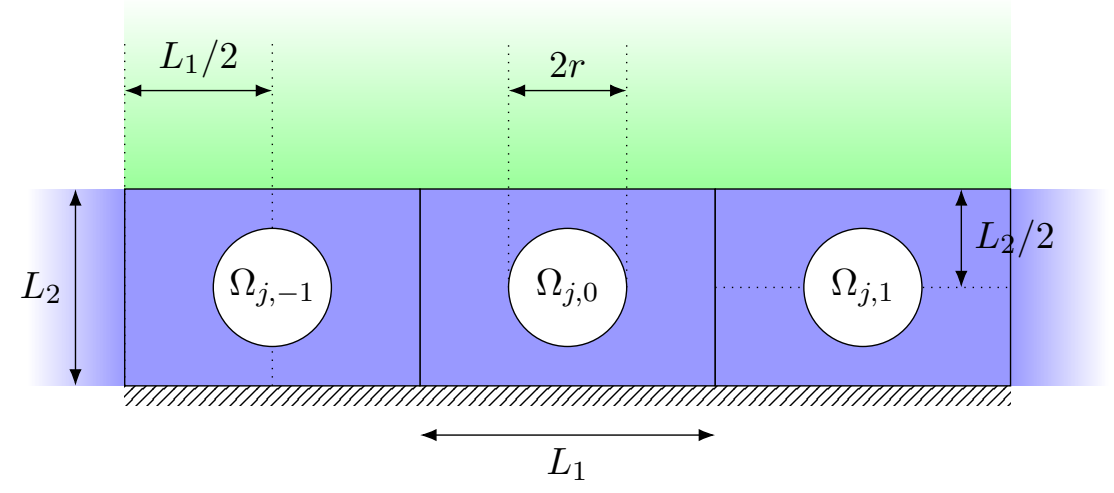

Figure 9. (Color online) Geometry of a one-dimensional periodic lattice with a cylindrical inclusion embedded in an isotropic homogeneous porous material.

The geometric parameters of the periodic array are $L_{1}=42 \mathrm{~mm}, L_{2}=25 \mathrm{~mm}$ and $r=9 \mathrm{~mm}$. The JCAL parameters of the homogeneous isotropic porous material are given in Tab. III. 
(a)

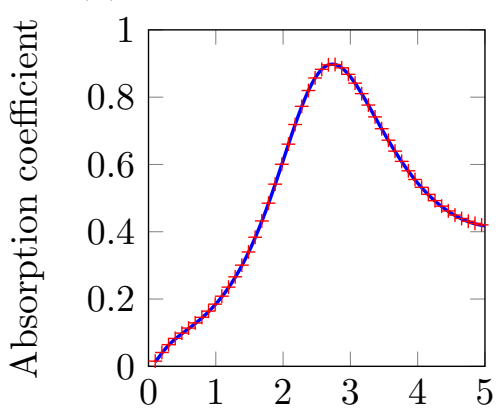

(c) Frequency $(\mathrm{kHz})$

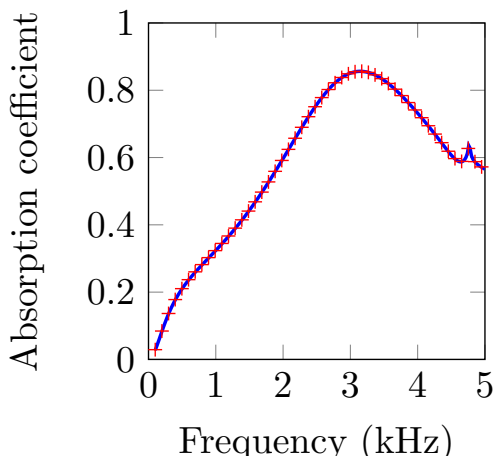

(b)

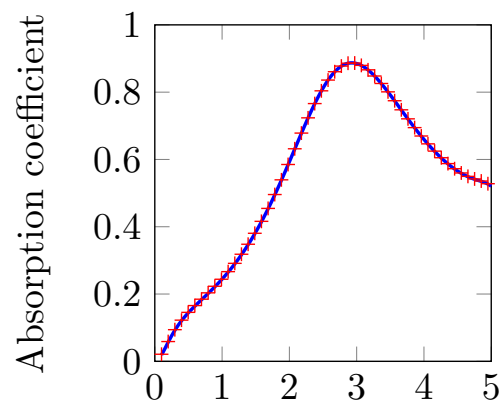

(d) Frequency $(\mathrm{kHz})$

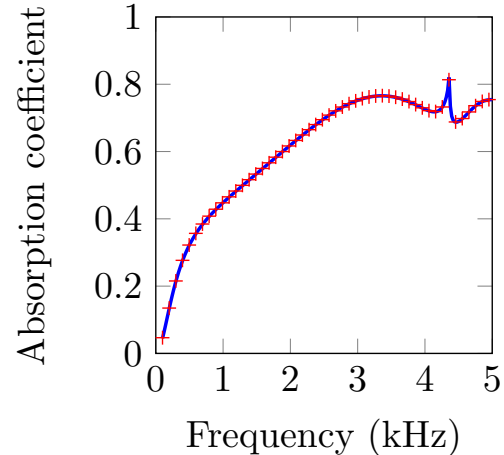

$\mathrm{MST}+\mathrm{FEM}$

Figure 10. (Color online) Comparison of FEM and MST results, for angles of incidence $\theta=\pi / 2$ (a), $\theta=\pi / 3(\mathrm{~b}), \theta=\pi / 4(\mathrm{c})$, and $\theta=\pi / 6(\mathrm{~d})$.

\begin{tabular}{cccccc}
\hline$\phi$ & $\Theta^{0}\left[\times 10^{-9} \mathrm{~m}^{2}\right]$ & $\tau^{\infty}$ & $\Lambda\left[\times 10^{-4} \mathrm{~m}\right]$ & $\Lambda^{\prime}\left[\times 10^{-4} \mathrm{~m}\right]$ & $K^{0}\left[\times 10^{-9} \mathrm{~m}^{2}\right]$ \\
\hline \hline 0.800 & 8.353 & 1.218 & 2.172 & 3.568 & 3.089 \\
\hline
\end{tabular}

Table III. JCAL parameters of the homogeneous isotropic porous material.

The results in Fig. 8 show the comparison of FEM and MST results for different angles of incidence. The results provided by the FEM and the MST are in excellent agreement, thus demonstrating the Wood anomaly at high frequencies, in Fig. 10(c,d). 


\section{ABBREVIATIONS}

- by order of appearance:

FDM Fused deposition modeling

FEM Finite element method

SRR Split-ring resonator

PLA Polylactic acid

STL Standard tessellation language

CNC Computer numerical control

JCAL Johnson-Champoux-Allard-Lafarge

PML Perfectly matched layer

SVD Singular value decomposition

TMM Transfer matrix method

MST Multiple scattering theory

\section{DATA AVAILABILITY}

The data that support the findings of this study are available from the corresponding author upon reasonable request.

\section{ACKNOWLEDGMENTS}

This research was funded by the Chaire Industrielle MACIA (ANR-16-CHIN-0002) funded by the Safran Group and the ANR (Agence Nationale de la Recherche, France).

\section{REFERENCES}

${ }^{1}$ J.-F. Allard and N. Atalla, Propagation of Sound in Porous Media: Modelling Sound Absorbing Materials (2nd ed.) (John Wiley \& Sons, Ltd, 2009).

${ }^{2}$ T. Cox and P. D'Antonio, Acoustic Absorbers and Diffusers: Theory, Design and Application (2nd ed.) (CRC Press, 2009).

${ }^{3}$ N. Jiménez, V. Romero-García, and J.-P. Groby, "Perfect absorption of sound by rigidlybacked high-porous materials," Acta Acustica united with Acustica 104, 396-409 (2018). 
${ }^{4}$ J. Boulvert, T. Cavalieri, J. Costa-Baptista, L. Schwan, V. Romero-García, G. Gabard, E. R. Fotsing, A. Ross, J. Mardjono, and J.-P. Groby, "Optimally graded porous material for broadband perfect absorption of sound," Journal of Applied Physics 126, 175101 (2019).

${ }^{5}$ T. Cavalieri, J. Boulvert, G. Gabard, V. Romero-García, M. Escouflaire, J. Regnard, and J.-P. Groby, "Graded and anisotropic porous materials for broadband and angular maximal acoustic absorption," Materials 13, 4605 (2020).

${ }^{6}$ N. Jiménez, W. Huang, V. Romero-García, V. Pagneux, and J.-P. Groby, "Ultra-thin metamaterial for perfect and quasi-omnidirectional sound absorption," Applied Physics Letters 109, 121902 (2016), https://doi.org/10.1063/1.4962328.

${ }^{7}$ V. Romero-García, G. Theocharis, O. Richoux, A. Merkel, V. Tournat, and V. Pagneux, "Perfect and broadband acoustic absorption by critically coupled sub-wavelength resonators," Sci Rep 6, 19519 (2016).

${ }^{8}$ J.-P. Groby, W. Huang, A. Lardeau, and Y. Aurégan, "The use of slow waves to design simple sound absorbing materials," Journal of Applied Physics 117, 124903 (2015), https://doi.org/10.1063/1.4915115.

${ }^{9}$ G. Ma, M. Yang, S. Xiao, Z. Yang, and P. Sheng, "Acoustic metasurface with hybrid resonances," Nature Mater 13, 873878 (2014).

${ }^{10}$ J. Christensen, V. Romero-García, R. Picó, A. Cebrecos, F. García de Abajo, N. Mortensen, M. Willatzen, and V. Sánchez-Morcillo, "Extraordinary absorption of sound in porous lamella-crystals," Sci Rep 4, 4674 (2014).

${ }^{11}$ K. Donda, Y. Zhu, S.-W. Fan, L. Cao, Y. Li, and B. Assouar, "Extreme low-frequency ultrathin acoustic absorbing metasurface," Applied Physics Letters 115, 173506 (2019), https://doi.org/10.1063/1.5122704.

${ }^{12}$ M. Yang, S. Chen, X. Fu, and P. Sheng, "Optimal sound-absorbing structures," Materials Horizons (2017).

${ }^{13}$ N. Jiménez, V. Romero-García, V. Pagneux, and J.-P. Groby, "Rainbow-trapping absorbers: Broadband, perfect and asymmetric sound absorption by subwavelength panels for transmission problems," Sci Rep 7, 13595 (2017).

${ }^{14}$ J.-P. Groby, O. Dazel, A. Duclos, L. Boeckx, and L. Kelders, "Enhancing the absorption coefficient of a backed rigid frame porous layer by embedding circular periodic inclusions," The Journal of the Acoustical Society of America 130, 3771-3780 (2011), 
https://doi.org/10.1121/1.3652865.

${ }^{15}$ C. Lagarrigue, J. P. Groby, V. Tournat, O. Dazel, and O. Umnova, "Absorption of sound by porous layers with embedded periodic arrays of resonant inclusions," The Journal of the Acoustical Society of America 134, 4670-4680 (2013), https://doi.org/10.1121/1.4824843. ${ }^{16}$ J.-P. Groby, C. Lagarrigue, B. Brouard, O. Dazel, V. Tournat, and B. Nennig, "Enhancing the absorption properties of acoustic porous plates by periodically embedding helmholtz resonators," The Journal of the Acoustical Society of America 137, 273-280 (2015), https://doi.org/10.1121/1.4904534.

${ }^{17}$ J.-P. Groby, C. Lagarrigue, B. Brouard, O. Dazel, V. Tournat, and B. Nennig, "Using simple shape three-dimensional rigid inclusions to enhance porous layer absorption," The Journal of the Acoustical Society of America 136, 1139-1148 (2014), https://doi.org/10.1121/1.4892760.

${ }^{18}$ J.-P. Groby, A. Wirgin, and E. Ogam, "Acoustic response of a periodic distribution of macroscopic inclusions within a rigid frame porous plate," Waves in Random and Complex Media 18, 409-433 (2008), https://doi.org/10.1080/17455030802061300.

${ }^{19}$ J.-P. Groby, A. Wirgin, L. De Ryck, W. Lauriks, R. P. Gilbert, and Y. S. Xu, "Acoustic response of a rigid-frame porous medium plate with a periodic set of inclusions," The Journal of the Acoustical Society of America 126, 685-693 (2009), https://doi.org/10.1121/1.3158936.

${ }^{20}$ E. Deckers, C. Claeys, O. Atak, J.-P. Groby, O. Dazel, and W. Desmet, "A wave based method to predict the absorption, reflection and transmission coefficient of two-dimensional rigid frame porous structures with periodic inclusions," Journal of Computational Physics $312,115-138$ (2016).

${ }^{21}$ Y. Zhou, D. Li, Y. Li, and T. Hao, "Perfect acoustic absorption by subwavelength metaporous composite," Applied Physics Letters 115, 093503 (2019), https://doi.org/10.1063/1.5107439.

${ }^{22}$ J. Yang, J. S. Lee, and Y. Y. Kim, "Metaporous layer to overcome the thickness constraint for broadband sound absorption," Journal of Applied Physics 117, 174903 (2015), https://doi.org/10.1063/1.4919844.

${ }^{23}$ L. Xiong, B. Nennig, Y. Aurégan, and W. Bi, "Sound attenuation optimization using metaporous materials tuned on exceptional points," The Journal of the Acoustical Society of America 142, 2288-2297 (2017), https://doi.org/10.1121/1.5007851. 
${ }^{24}$ J.-P. Groby, A. Duclos, O. Dazel, L. Boeckx, and W. Lauriks, "Absorption of a rigid frame porous layer with periodic circular inclusions backed by a periodic grating," The Journal of the Acoustical Society of America 129, 3035-3046 (2011), https://doi.org/10.1121/1.3561664.

${ }^{25}$ T. Weisser, J.-P. Groby, O. Dazel, F. Gaultier, E. Deckers, S. Futatsugi, and L. Monteiro, "Acoustic behavior of a rigidly backed poroelastic layer with periodic resonant inclusions by a multiple scattering approach," The Journal of the Acoustical Society of America 139, 617-629 (2016), https://doi.org/10.1121/1.4940669.

${ }^{26}$ J.-L. Auriault, C. Boutin, and C. Geindreau, Homogenization of coupled phenomena in heterogenous media (ISTE; J. Wiley, 2009).

${ }^{27}$ E. Sanchez-Palencia, Non-homogeneous media and vibration theory, Lecture notes in physics No. 127 (Springer, 1980) OCLC: 6447016.

${ }^{28}$ A. Bensoussan, J.-L. Lions, and G. Papanicolaou, Asymptotic Analysis of Periodic Structures, Vol. 5 (North-Holland Publishing Company, 1978).

${ }^{29}$ J. A. Nelder and R. Mead, "A simplex method for function minimization," The Computer Journal 7, 308-313 (1965).

${ }^{30}$ W. H. Press, S. A. Teukolsky, W. T. Vetterling, and B. P. Flannery, "Numerical recipes: the art of scientific computing," (2007), OCLC: ocn123285342.

${ }^{31}$ J. Boulvert, J. Costa-Baptista, T. Cavalieri, M. Perna, E. Fotsing, V. Romero-García, G. Gabard, A. Ross, J. Mardjono, and J.-P. Groby, "Acoustic modeling of micro-lattices obtained by additive manufacturing," (2020).

${ }^{32}$ T. G. Zieliski, K. C. Opiela, P. Pawowski, N. Dauchez, T. Boutin, J. Kennedy, D. Trimble, H. Rice, B. Van Damme, G. Hannema, R. Wrbel, S. Kim, S. Ghaffari Mosanenzadeh, N. X. Fang, J. Yang, B. Briere de La Hosseraye, M. C. Hornikx, E. Salze, M.-A. Galland, R. Boonen, A. Carvalho de Sousa, E. Deckers, M. Gaborit, and J.-P. Groby, "Reproducibility of sound-absorbing periodic porous materials using additive manufacturing technologies: Round robin study," Additive Manufacturing 36, 101564 (2020).

${ }^{33}$ W. Huang, L. Schwan, V. Romero-Garca, J.-M. Gnevaux, and J.-P. Groby, "3d-printed sound absorbing metafluid inspired by cereal straws," Sci Rep 9, 8496 (2019).

${ }^{34}$ D. Lafarge, P. Lemarinier, J. F. Allard, and V. Tarnow, "Dynamic compressibility of air in porous structures at audible frequencies," 102, 1995-2006 (1997). 
${ }^{35}$ T. Cavalieri, J. Boulvert, L. Schwan, G. Gabard, V. Romero-Garca, J.-P. Groby, M. Escouflaire, and J. Mardjono, "Acoustic wave propagation in effective graded fully anisotropic fluid layers," The Journal of the Acoustical Society of America 146, 3400-3408 (2019).

${ }^{36}$ A. Terroir, L. Schwan, T. Cavalieri, V. Romero-García, G. Gabard, and J.-P. Groby, "General method to retrieve all effective acoustic properties of fully-anisotropic fluid materials in three dimensional space," Journal of Applied Physics 125, 025114 (2019).

${ }^{37}$ T. G. Zieliński, R. Venegas, C. Perrot, M. Červenka, F. Chevillotte, and K. Attenborough, "Benchmarks for microstructure-based modelling of sound absorbing rigid-frame porous media," Journal of Sound and Vibration 483, 115441 (2020).

${ }^{38}$ M. Niskanen, J.-P. Groby, A. Duclos, O. Dazel, J. C. Le Roux, N. Poulain, T. Huttunen, and T. Lhivaara, "Deterministic and statistical characterization of rigid frame porous materials from impedance tube measurements," The Journal of the Acoustical Society of America 142, 2407-2418 (2017).

${ }^{39}$ A. Sommerfeld, Partial differential equations in physics, Lectures on theoretical physics No. by Arnold Sommerfeld ; 6 (Acad. Pr, 1964) OCLC: 250848097.

${ }^{40}$ S. H. Schot, "Eighty years of sommerfeld's radiation condition," Historia Mathematica 19, 385-401 (1992).

${ }^{41}$ J.-P. Berenger, "A perfectly matched layer for the absorption of electromagnetic waves," Journal of Computational Physics 114, 185-200 (1994).

${ }^{42}$ M. R. Stinson, "The propagation of plane sound waves in narrow and wide circular tubes, and generalization to uniform tubes of arbitrary crosssectional shape," The Journal of the Acoustical Society of America 89, 550-558 (1991), https://doi.org/10.1121/1.400379.

${ }^{43}$ J.-P. Groby, W. Lauriks, and T. E. Vigran, "Total absorption peak by use of a rigid frame porous layer backed by a rigid multi-irregularities grating," The Journal of the Acoustical Society of America 127, 2865-2874 (2010), https://doi.org/10.1121/1.3337235.

${ }^{44}$ A. Dresden, "The fourteenth western meeting of the american mathematical society," Bull. Amer. Math. Soc. 26, 385-397 (1920).

${ }^{45}$ R. Penrose, "A generalized inverse for matrices," Math. Proc. Camb. Phil. Soc. 51, 406-413 (1955).

${ }^{46}$ B. Brouard, D. Lafarge, and J.-F. Allard, "A general method of modelling sound propagation in layered media," 183, 129-142 (1995). 
${ }^{47}$ M. C. Pease, Methods of Matrix Algebra. (Elsevier NetLibrary, Incorporated [distributor, 1965) OCLC: 944502142.

${ }^{48}$ R. A. Horn and C. R. Johnson, Matrix analysis, second edition, corrected reprint ed. (Cambridge University Press, New York, NY, 2017).

${ }^{49}$ V. Romero-García and A.-C. Hladky-Hennion, Fundamentals and Applications of Acoustic Metamaterials (2019) p. 325. 